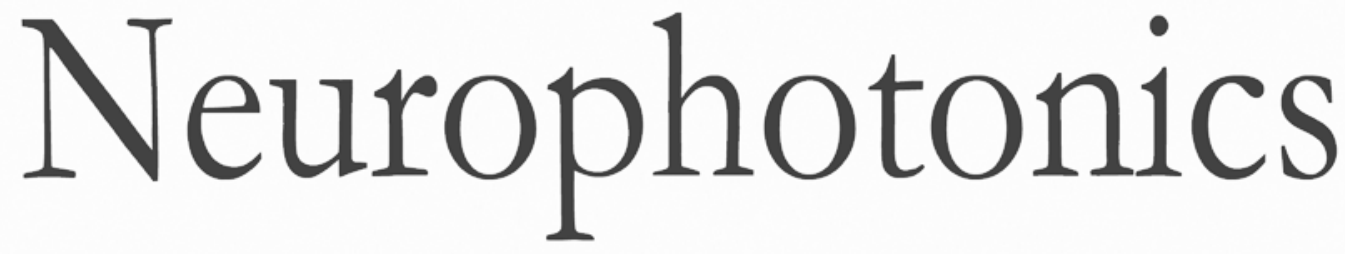

\title{
Multiple speckle exposure imaging for the study of blood flow changes induced by functional activation of barrel cortex and olfactory bulb in mice
}

Haleh Soleimanzad

François Smekens

Juliette Peyronnet

Marjorie Juchaux

Olivier Lefebvre

David Bouville

Christophe Magnan

Hirac Gurden

Frederic Pain 


\title{
Multiple speckle exposure imaging for the study of blood flow changes induced by functional activation of barrel cortex and olfactory bulb in mice
}

\author{
Haleh Soleimanzad, ${ }^{a, b}$ François Smekens, ${ }^{a}$ Juliette Peyronnet, ${ }^{a}$ Marjorie Juchaux, ${ }^{\text {a,c }}$ Olivier Lefebvre, ${ }^{a}$ \\ David Bouville, ${ }^{\mathrm{c}}$ Christophe Magnan, ${ }^{\mathrm{b}}$ Hirac Gurden, ${ }^{\mathrm{b}}$ and Frederic Pain ${ }^{\mathrm{a}, *}$ \\ aIMNC, CNRS, Université Paris-Sud, Université Paris-Saclay, Orsay, France \\ bBFA, CNRS, Université Paris Diderot, Paris, France \\ ${ }^{\circ} \mathrm{C} 2 \mathrm{~N}$, CNRS, Université Paris-Sud, Université Paris-Saclay, Palaiseau, France
}

\begin{abstract}
Speckle contrast imaging allows in vivo imaging of relative blood flow changes. Multiple exposure speckle imaging (MESI) is more accurate than the standard single-exposure method since it allows separating the contribution of the static and moving scatters of the recorded speckle patterns. MESI requires experimental validation on phantoms prior to in vivo experiments to ensure the proper calibration of the system and the robustness of the model. The data analysis relies on the calculation of the speckle contrast for each exposure and a subsequent nonlinear fit to the MESI model to extract the scatterers correlation time and the relative contribution of moving scatters. We have designed two multichannel polydimethylsiloxane chips to study the influence of multiple and static scattering on the accuracy of MESI quantitation. We also propose a method based on standard $\mathrm{C}++$ libraries to implement a computationally efficient analysis of the MESI data. Finally, the system was used to obtain in vivo hemodynamic data on two distinct sensory areas of the mice brain: the barrel cortex and the olfactory bulb. ( The Authors. Published by SPIE under a Creative Commons Attribution 4.0 Unported License. Distribution or reproduction of this work in whole or in part requires full attribution of the original publication, including its DOI. [DOI: 10.1117/1.NPh.6.1.015008]

Keywords: speckle contrast imaging; multiple exposure imaging; blood flow; functional imaging; barrel cortex; olfactory bulb.

Paper 18066R received Nov. 5, 2018; accepted for publication Feb. 13, 2019; published online Mar. 5, 2019.
\end{abstract}

\section{Introduction}

Speckle contrast imaging has been developed during the last decades as a powerful tool for blood flow mapping, particularly in rodent brain structures. ${ }^{1-3}$ The technique relies on the statistical properties of dynamic speckle patterns observed when a coherent light source is used to illuminate biological tissues, like the eye, ${ }^{4-6}$ or the exposed cortical tissues of the brain. ${ }^{7-12}$ In its simplest implementation, it only requires a standard video camera and a single-mode laser. ${ }^{13,14}$ The speckle contrast is computed over a spatial or temporal ensemble of pixels. As shown in Eq. (1), contrast $K$ is defined as the ratio of the standard deviation of intensities above the mean of intensities of the pixels in the ensemble. If the scatterers are mobile, the contrast $K$ is related to the exposure time $T$. Basically, moving scatterers create dynamic speckle patterns that are averaged during the exposure time $T$. Areas in the image where scatterers are moving fast will appear blurred, producing locally a lower contrast. Theoretically, the values of $K$ range from 0 for fast moving scatterers up to 1 for purely static scatterers:

$K(T)=\frac{\sigma(T)}{\langle I(T)\rangle}$.

The local speckle contrast $K$ is calculated using spatial, ${ }^{4,7,15}$ temporal, ${ }^{5,8,16-18}$ or spatiotemporal ${ }^{19}$ sliding windows. Spatial calculation of $K$ allows studying dynamic phenomenon with a temporal resolution equal to the frame rate of image capture but at the expense of a decreased spatial resolution. The temporal

*Address all correspondence to Frederic Pain, E-mail: frederic.pain @u-psud.fr implementation provides a spatial resolution restricted only by the optics and the sensor's pixel dimension but with a limited temporal resolution since data are averaged in the temporal dimension. Theory of speckle formation relates the speckle contrast to the autocovariance of the intensities fluctuations of an individual speckle. ${ }^{20}$ Equation (2) provides the relation between the contrast $K$, the exposure time $T$, and the scatterers correlation time $\tau_{c}$. The variable $x$ is equal to $T / \tau_{c} ; \rho$ is defined as the ratio of the intensity contribution of mobile scatterers over the total intensity due to mobile and static scatterers; $\beta$ is a dimensionless constant that accounts for spatial averaging of the speckle grains and instrumental parameters:

$K\left(T, \tau_{c}\right)=\left(\beta \frac{e^{-2 x}-1+2 x}{2 x^{2}}\right)^{\frac{1}{2}}$.

Under the assumption of single-dynamic scattering, the blood velocity is inversely proportional to $\tau_{c}$ :

$v \propto \frac{1}{\tau_{c}}$

Yet the exact relation between $\tau_{c}$ and the velocity is not known due to the lack of information about the actual geometrical of scatterers, the assumption of a Lorentzian velocity distribution of scatterers, and the contribution of distinct flows to the speckle patterns. Previous analysis has concluded that absolute quantitation of cerebral blood flow (CBF) using singleexposure speckle analysis is difficult to obtain due to inherent hypotheses of the model, the difficulty of measuring the instrumental parameter $\beta$, and ultimately the difficulty of evaluating 
the contribution of static scatterers to the speckle patterns. ${ }^{21,22}$ A model of speckle contrast $K$ was derived successfully in order to account for the contribution of the static scatterers and provide a more accurate derivation of the correlation time $\tau_{c}:{ }^{21}$

$K\left(T, \tau_{c}\right)=\left[\beta \rho^{2} \frac{e^{-2 x}-1+2 x}{2 x^{2}}+4 \beta \rho(1-\rho) \frac{e^{-x}-1+x}{x^{2}}+v_{s}\right]^{\frac{1}{2}}$.

The term $v_{s}$ pools the contributions to $K$ due to image noise and nonergodicity. The first goal of our study is to evaluate several normalizations approaches proposed to account for multiple scattering events not considered in the model and that may happen in large vessels due to the high-scattering optical coefficient of blood. We have designed microfluidic chips to experimentally study the effect of the vessel size on the accuracy of flow retrieval. Fluid with different scatterers concentration has been used to evaluate the impact of optical scattering in vessels. A major challenge for multiple exposure speckle imaging (MESI) imaging is the development of a simple and efficient analysis workflow. In our set up, MESI acquisition generates about 2 GB of data for a 40-s recording (image size of $700 \times$ 500 pixels $\times 16$ bits). The processing of the data is a very timeconsuming operation to generate the corresponding contrast frames and the subsequent pixel-wise fit to Eq. (4) of a stack of contrast frames acquired at different exposure times. We have implemented an optimized contrast calculation and fit procedure using open-source $\mathrm{C}++$ libraries resulting in a simple, yet efficient analysis method of MESI data. The second goal of our study is to optimize the number of exposure times used in order to obtain a temporal resolution adequate for the study of brain hemodynamic changes. Finally, MESI imaging was applied in vivo in anesthetized mice to evaluate the practicability of the technique to image CBF through the skull. MESI imaging of the barrel cortex $(\mathrm{BC})$ in the somatosensory cortex was compared to data recorded in the olfactory bulb (OB) both at rest and during functional activation.

\section{Materials and Methods}

\subsection{MESI Set Up}

The architecture of the MESI set up is shown in Fig. 1(a). The system relies on an sCMOS flash camera (Hamamatsu, Massy, France) camera with a fixed exposure time to capture images of the brain tissues illuminated with a continuous laser diode modulated in duration and intensity $(633 \mathrm{~nm}, 200 \mathrm{~mW}$, SGL, Shanghai, China). The fast modulation of the laser is obtained using an acousto-optic modulator (AOM) (AA Optoelectronics, Orsay, France) driven by a high-frequency signal modulated by an arbitrary waveform generator (Keysight Series 33600A, Malaysia). The AOM is devoid of mechanical parts thus allowing very fast and reproducible operation. It relies on diffraction through a controlled phase grating to modulate the first diffraction order of the signal. A fixed aperture diaphragm is used to filter the nondiffracted light as well as the higher diffraction orders. The imaging lens is a custom-built dual lenses macroscope ${ }^{23}$ allowing a numerical aperture of 0.125 for a magnification factor of 1.6 and a working distance of $1.5 \mathrm{~cm}$. The exposure time of the camera is fixed while the laser pulses are modulated in time and intensity resulting in changes of the actual exposure time while maintaining the intensity and consequently the shot noise constant. The complete set up was described in details previously. ${ }^{24}$ (a)

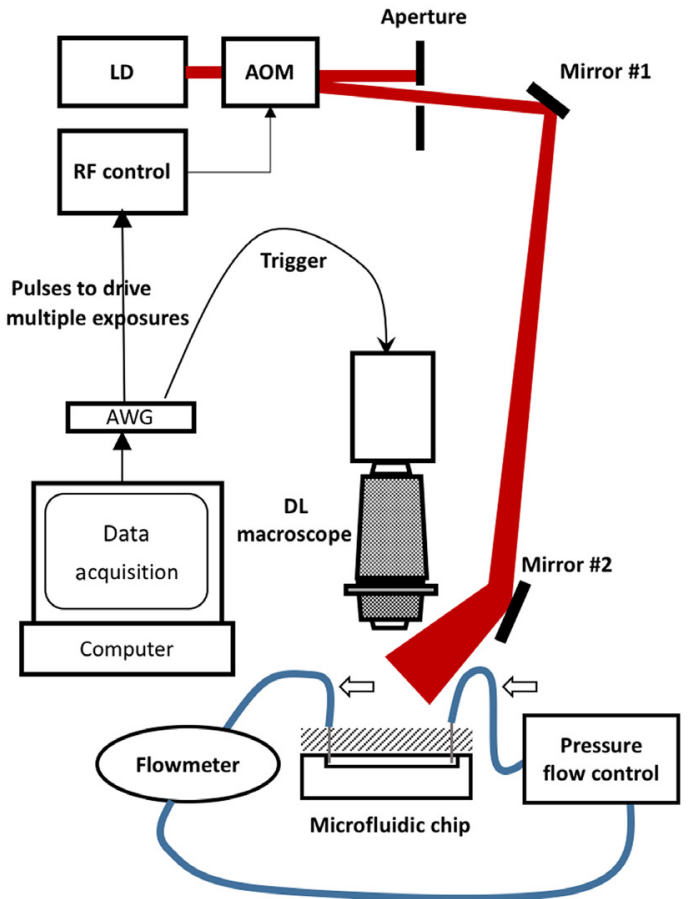

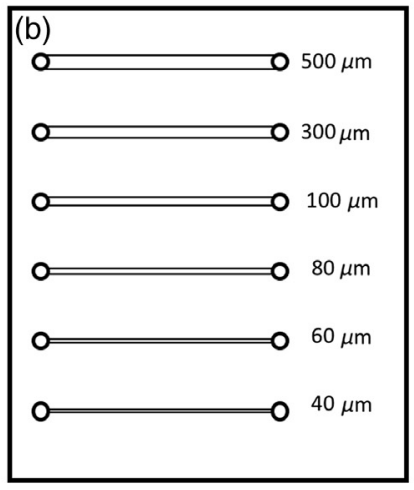

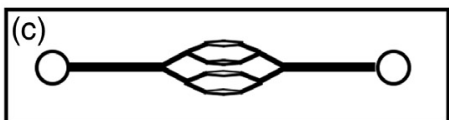

Fig. 1 Experimental set up: (a) MESI set up. LD, laser diode; AOM, acousto-optic modulator; DLM, dual lens macroscope; AWG, arbitrary waveform generator; RF, radiofrequency control. (b) Microfluidic chip\#1 with single channels and (c) microfluidic chip\#2 with channel arborescence. 


\subsection{MESI Data Analysis}

Contrarily to the single-exposure speckle imaging method, the estimation of the correlation time $\tau_{c}$ of a multiexposure speckle acquisition is a computationally intensive task. The ultimate goal of the data analysis is to obtain 2-D maps of $1 / \tau_{c}$, named inverse correlation time (ICT) maps. Thus it is possible to calculate relative changes in ICT for different physiological or pathological conditions or during functional activation. The data analysis method is based on Eqs. (2)-(4). ${ }^{21}$ We propose here an optimized procedure to obtain ICT maps from multiple exposure speckle contrast images.

Prior to the fit to the MESI models, the raw gray level images were processed to obtain the speckle contrast images. Four different approaches using sliding windows or cuboid of pixels were evaluated to calculate the contrast images. The spatial contrast $K_{\mathrm{s}}$ was calculated with a spatial window of $5 \times 5$ pixels in the same frame, the temporal contrast $K_{\mathrm{t}}$ was calculated using a single pixel in 15 consecutive images $(1 \times 15$ window $)$. For the spatiotemporal $K_{\text {st }}$ approach, the contrast is calculated for individual a $5 \times 5$ spatial window and then average temporally for five consecutive frames. The process is reverse for temporospatial contrast $K_{\mathrm{ts}}$. The detailed definition and calculation for each contrast have been defined previously. ${ }^{25}$ In the presented data except when explicitly mentioned, the speckle contrast was calculated as $K_{\text {st }}$.

The analysis program was written in $\mathrm{C}++$ and used multiCPU parallelizing (Fig. 2) to reduce the analysis time compared to classical MATLAB or Python approaches. For the sake of efficiency, all the manipulation on images (read, save, extract the values, etc.) were performed using the ITK library (Insight Segmentation and Registration Toolkit ${ }^{26}$ ). In addition to its speed, this framework has the advantage to be an open-source $\mathrm{C}++$ library dedicated to the medical image processing and can handle a large variety of file formats. The data analysis workflow is shown in Fig. 2. The input speckle contrast image is a single-binary (.raw) file with $N_{f}$ frames. These frames are composed of $S$ multiexposure stacks, each composed of $t$ images acquired with increasing exposure times $\left(N_{f}=S \times t\right)$. The first step of the procedure consists in splitting the input file into $S$-independent stacks, which are then processed simultaneously on separated CPU cores (Intel ${ }^{\circledR}$ Xeon ${ }^{\circledR}$ CPU E5-2690 $3 \mathrm{GHz}$ with 40 cores). For each pixel of a stack, we estimate the value of $\beta$ by fitting the $t$ exposure times with Eq. (2). The resulting value is then used as a fixed term for the second fit with Eq. (4), evaluating the values of $\tau_{c}, \rho$, and $v_{s}$. For both fits, we used the Levenberg-Marquardt (LM) algorithm proposed in the ALGLIB Free Edition C++ library. ${ }^{27}$ Although ITK library also includes the LM algorithm, the version implemented in ALGLIB can scale and constrain the fitted parameter, making the optimization faster and the optimized values physically relevant. The last step of the procedure was the creation of the contrast parameters images, i.e., $\beta, \rho, v$, and $1 / \tau_{c}$ images. The constraints, scaling values, and initial values chosen to estimate the parameters for both fits are provided in Table 1.

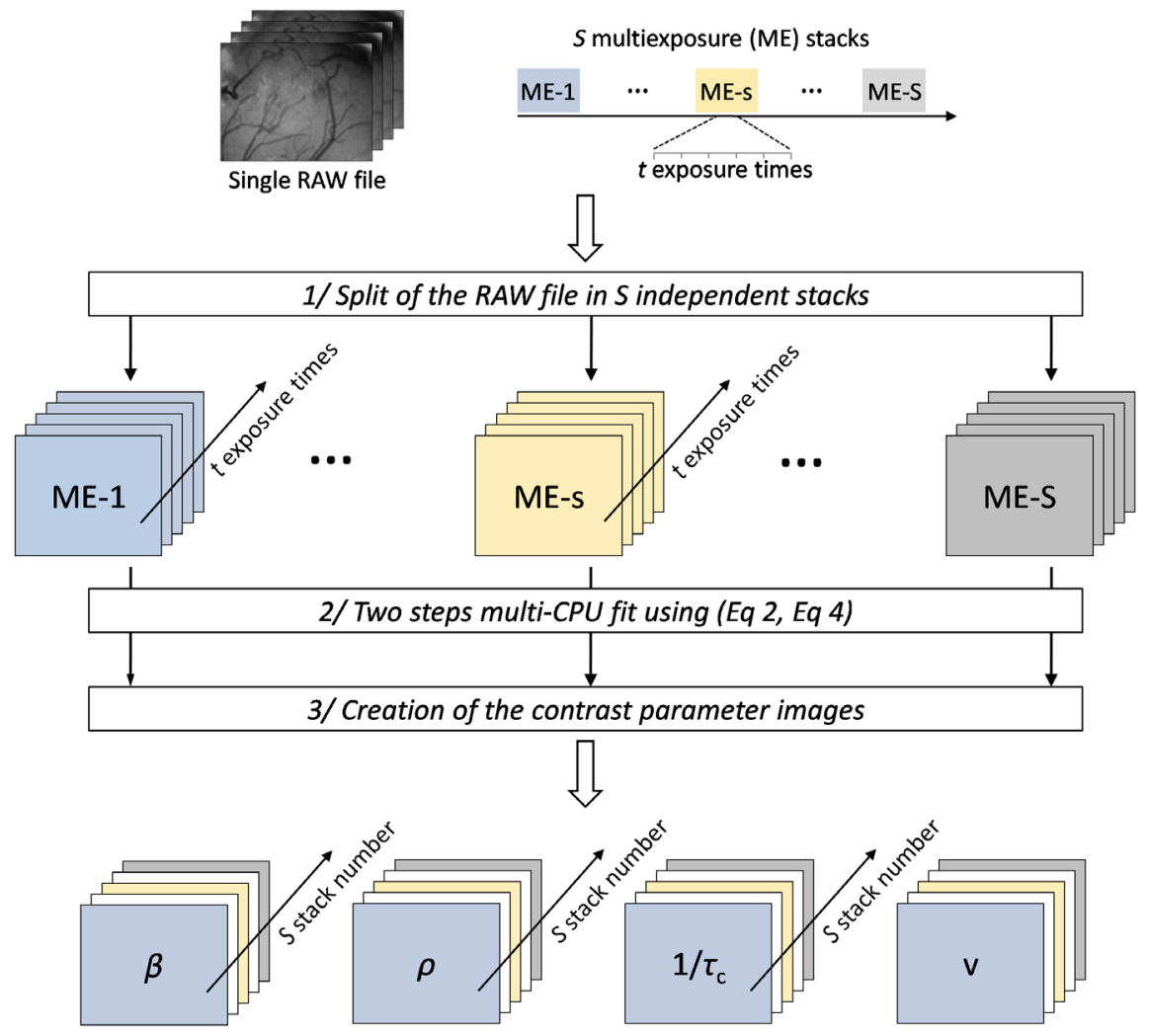

Fig. 2 Scheme of the speckle contrast data analysis procedure. A single file of the computed speckle contrast frames corresponding to the full acquisition is split into $S$ multiexposure stacks each composed of $t$ exposure times. Two fits are successively applied on each pixels of each stack. Finally, the estimated speckle contrast parameters are grouped as separated images stacks. 
Table 1 Parameterization of the LM algorithm used for the data analysis.

\begin{tabular}{lcccc} 
Parameter & $\beta$ & $\tau_{c}$ & $v$ & $\rho$ \\
\hline $\begin{array}{l}\text { Initial_first fit } \\
\text { [Eq. (2)] }\end{array}$ & 0.05 & 10.0 & 0.001 & - \\
$\begin{array}{l}\text { Initial_second fit } \\
\text { [Eq. (4)] }\end{array}$ & $\begin{array}{c}\text { Fixed } \\
\text { (first fit) }\end{array}$ & $\begin{array}{c}\text { First fit } \\
\text { result }\end{array}$ & $\begin{array}{c}\text { First fit } \\
\text { result }\end{array}$ & 0.5 \\
Constraint & {$[0.0,+\infty]$} & {$[0.0,20.0]$} & {$[0.0,0.5]$} & {$[0.0,1.0]$} \\
Scale & 0.05 & 10.0 & 0.001 & 0.5 \\
\hline
\end{tabular}

\subsection{Microfluidic Phantom Design and Implementation}

The microfluidic chips were manufactured using standard photolithography techniques. A photoresist (SU-8 3025, MicroChem) with a controlled thickness was used to create microstructures on a silicon wafer. A mixture of polydimethylsiloxane (PDMS) prepolymer and curing agent was prepared (Sylgard 184 Silicone Elastomer Kit, Neyco, France). It was mixed and degassed before being poured on the silicon mold and heated at $85^{\circ} \mathrm{C}$ to cure for $1 \mathrm{~h}$. After curing, the PDMS was carefully peeled from the mold. Holes for tubing connection of the chip were punched through the PDMS. A PDMS layer with a thickness of $300 \mu \mathrm{m}$ was used to seal the chip. Sealing was obtained either using oxygen plasma or corona discharge (Corona SB, Blackholelabs, Paris, France). The flow in the chips was controlled using a pressure controller (MFCS, Maesflo System Fluigent, Kremlin Bicêtre, France) with a feedback from a flow monitor (S and XS models, Fluigent, Kremlin Bicêtre, France) allowing a more stable, fast, and accurate control than syringe pumps classically used. Intralipid (I141, Sigma Aldrich) solution was used a flowing fluid. For most experiments, it was diluted to $2 \%$ to obtain optical scattering properties representative of whole blood.

Chip \#1 [Fig. 1(b)] is composed of single channels with increasing width ranging from 40 up to $500 \mu \mathrm{m}$. The height and length of all channels are $75 \mu \mathrm{m}$ and $1 \mathrm{~cm}$, respectively. Chip \#2 [Fig. 1(c)] is composed of an arborescence of channels of 200 , 50,20 , and $10 \mu \mathrm{m}$ with respective lengths of $6,0.5,0.4$, and $0.2 \mathrm{~mm}$. In order to evaluate the ability of the MESI technique to remove the contribution of static scatterers due to a skull layer, the PDMS cover used to seal this chip was either composed of clear PDMS or PDMS with an amount of $\mathrm{TiO}_{2}{ }^{28}$ The reduced optical scattering coefficients of the PDMS covers were measured using the well-established integrating sphere method. ${ }^{29}$ The concentration of $\mathrm{TiO}_{2}$ in the covers of chip \#2 was adjusted to mimic the reduced optical scattering coefficients previously measured in mice skulls. ${ }^{30}$ The reduced scattering coefficients of the PDMS covers were $0 \mathrm{~mm}^{-1}$ (chip \#2a no $\mathrm{TiO}_{2}$, pure transparent PDMS), $1 \mathrm{~mm}^{-1}$ (chip \#2b $1.91 \mathrm{mg} / \mathrm{ml}$ of $\mathrm{TiO}_{2}$ ), and $3 \mathrm{~mm}^{-1}$ (chip \#2c $2.80 \mathrm{mg} / \mathrm{ml}$ of $\mathrm{TiO}_{2}$ ), which corresponds to the reduced scattering coefficient of mice skull. ${ }^{30}$ Imaging chip \#2a mimics imaging through an open cranial window with a layer of transparent agarose above the tissue, whereas chip \#2b has an intermediate reduced scattering coefficient corresponding to a thinned skull preparation. Three sets of experiments were performed on the chips. First, using chip \#1, we have studied the accuracy of the MESI technique as a function of the channel width for a physiological range of flows (from 1 to $7 \mu \mathrm{l} / \mathrm{min}$ ) of $2 \%$ Intralipid. Second, the impact on flow retrieval of the different concentrations of flowing scatterers was studied using Intralipid solutions with concentrations of $2 \%, 4 \%, 10 \%$, and $20 \%$. Finally, using chip \#2a,b,c, the method used to calculate the speckle contrast and the impact of static scatterers in the configuration of through-skull imaging were studied.

\subsection{In Vivo Experiments}

All experiments were performed in accordance with the European Directive 86/609/EEC regarding the care and use of laboratory animals. In vivo MESI recordings were carried out in anesthetized mice through skull and after a craniotomy was performed over the OB or the BC. 4 to 8 weeks-old C57BL6 male mice ( $n=6,15$ to $33 \mathrm{gr}$ ) were anesthetized with a cocktail of ketamine $(100 \mathrm{mg} / \mathrm{kg}$ Imalgen $)$ and metedomidine $(0.5 \mathrm{mg} / \mathrm{kg})$, injected intraperitoneally. The anesthesia level was adjusted if necessary throughout the experiment. Surgery has begun when the mouse no longer responds to hindpaw pinch. During the entire experiment, the animal was placed on a thermostatically controlled heating pad. Body temperature was maintained rectally at $37^{\circ} \mathrm{C}$. After securely placed in a stereotaxic, imaging was carried out over regions of about $3 \times 3 \mathrm{~mm}^{2}$ and $1.5 \times 1.5 \mathrm{~mm}^{2}$ over the $\mathrm{BC}$ and $\mathrm{OB}$, respectively. These regions were exposed first without a craniotomy (keeping skull intact, without thinning) and second after a skull opening was performed over the $\mathrm{BC}$ and the $\mathrm{OB}$. A low-temperature agarose layer $(1.2 \%)$ was placed over dura to minimize brain movements. Sensory stimulations were carried out using: (i) a custom-designed olfactometer for $\mathrm{OB}$ and (ii) a custom-designed mechanical stimulator for whiskers. In both cases, stimulation was synchronized to the MESI acquisition. An olfactory sensory simulation trial consisted of $12 \mathrm{~s}$ of baseline (with airflow), $12 \mathrm{~s}$ of odorant stimulation, and $24 \mathrm{~s}$ of airflow clean-up. One experiment consisted in up to five trials recordings for olfactory stimulations with hexanal $5 \%$ or isoamyl acetate $5 \%$ diluted in mineral oil. Stimulations with two odorants were interleaved randomly to prevent habituation. A somatosensory stimulation consisted of $70 \mathrm{~s}$ of data acquisition at $5 \mathrm{~Hz}$ and an oscillation amplitude of $7 \mathrm{~mm}$, with $60 \mathrm{~s}$ stimuli starting after $10 \mathrm{~s}$ considering as a baseline. This protocol was repeated 3 times for each stimulus. The detailed craniotomy procedures and the custom olfactometer set up were described previously. ${ }^{31}$

\section{Results and Discussion}

\subsection{Impact of the Vessel Diameter and Mobile Scatterers Concentration on the ICT Values}

The knowledge of the microfluidic channels dimensions in chip \#1 as well as the ability to set the optical scattering properties of the flowing fluid allowed the experimental study of the robustness of previously proposed normalization techniques to account for multiple scattering effects. ${ }^{2,32}$ Indeed, the mathematical models that support the derivation of a physiologically meaningful parameter (ICT) assume that the collected light has undergone a single-dynamic scattering event. This may be true in small capillaries but due to the highly optical scattering coefficient of blood this assumption breaks down in larger vessels. It has been shown from the dynamic light scattering theory that speckle correlation time is weighted by the number of scattering events in the multiple scattering regime. ${ }^{33}$ The analysis of 
speckle data was carried out over a range of configuration with physiologically relevant channel diameters and flows. We have studied the effect of the vessel's diameter and the effect of the scatterers concentration. Figure 3(a) presents the ICT as a function of the flow for channels with increasing widths. For all channels, the ICT derived from the MESI model increases roughly linearly with the flow. However, flows higher than $1 \mu \mathrm{l} / \mathrm{min}$ lead to significant differences in the ICT values depending on the channel width. The differences in ICTs are stronger as the flow increases, and the ICT values are lower for wider channels. Figure 3(b) shows the effect of the normalization of ICT by the characteristic length $L_{c}$ of the channels as proposed previously. ${ }^{34}$ Here, as the vessels are not cylindrical but parallepipedic, we have calculated $L_{c}$ for each channel as the square root of its depth is multiplied by its width. As can be seen, this normalization provides a metric of the flow that is consistent for flows up to $4 \mu \mathrm{l} / \mathrm{min}$ for the considered vessels. However, the normalization is less robust for higher flows and fails for all flows in larger vessels of 300 and $500 \mu \mathrm{m}$ width (Fig. 4). The vessels' dimensions and flows observed in laser speckle contrast imaging of the cortical vasculature of small animals models are within the limits of accurate normalization. However these limits become a significant issue if the speckle imaging technique is used in a clinical per-operative context. ${ }^{35}$

The hypothesis of a single-dynamic scattering event for photons that contribute to the speckle patterns is also highly dependent on the scattering properties of the flowing fluid. Increasing the concentration of scatterers increases the number of scattering events and in turn modifies the speckle patterns. Here, we focused on the physiological range of the optical scattering properties of blood, which are related to the hematocrit (i.e., the relative proportion of red blood cells in plasma). Considering physiological hematocrit changes of about $\pm 20 \%$ around a baseline value of $47 \%,{ }^{36}$ the reduced scattering coefficient of blood is likely to vary between 1.5 and $2.6 \mathrm{~mm}^{-1} .{ }^{36}$ As the reduced scattering coefficient of Intralipid solution scales linearly with concentration, ${ }^{37}$ the flowing solution concentration can be adjusted to mimic the reduced scattering of blood. A solution with Intralipid concentration of $2 \%$ is assumed to simulate reasonably the reduced scattering properties of normal blood. ${ }^{34,38}$ In our experiments, doubling the concentration of the scatterers leads to an increase of the calculated ICT for all flows in a $60-\mu \mathrm{m}$ width channel [Fig. 5(a)].

In Fig. 5(b), the data have been normalized by the reduced scattering coefficient of the flowing fluid and by the characteristic length of the channel as proposed recently. ${ }^{32,34}$ The normalized data show a good agreement for flows up to $3 \mu \mathrm{l} / \mathrm{min}$ for both scatterers concentrations. For higher flows, the normalization does not efficiently retrieve the same ICT for identical flows. Similar results are observed in smaller and larger channels (data not shown). Interestingly, for all channels, high concentrations of scatterers (solution of Intralipid 10\% and 20\%) lead to a "saturation" of ICT values as can be seen in Fig. 6 for channels widths of 60 and $300 \mu \mathrm{m}$. For both channels, $10 \%$ and $20 \%$ Intralipid solutions lead to identical ICT values for different flows before any normalization. This suggests that the mean number of scattering events undergone by each photon
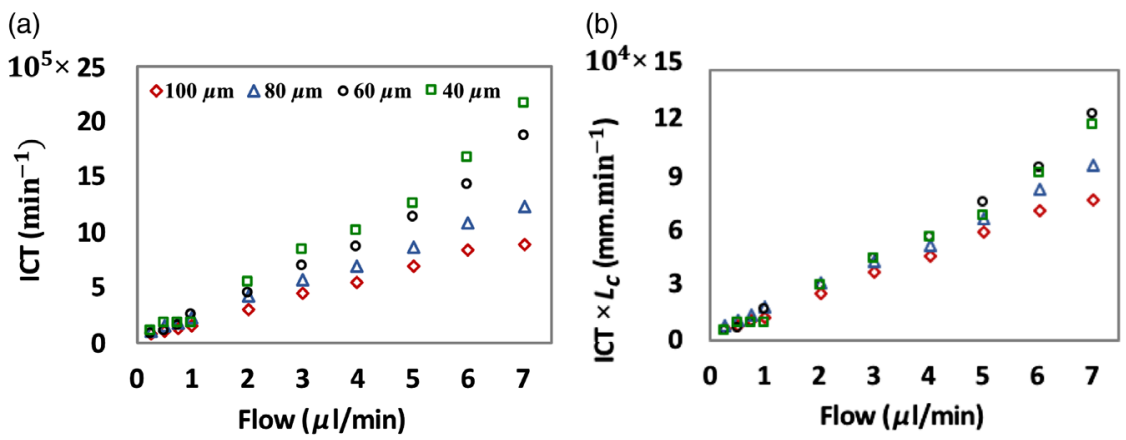

Fig. 3 Impact of the normalization of multiple dynamic scattering by the characteristic dimension of the channels. Data have been obtained by flowing $2 \%$ Intralipid solution into the channels of chip\# 1 . (a) Raw ICT and (b) ICT normalized by the characteristic dimension $L_{c}$ of each channel.

(a)

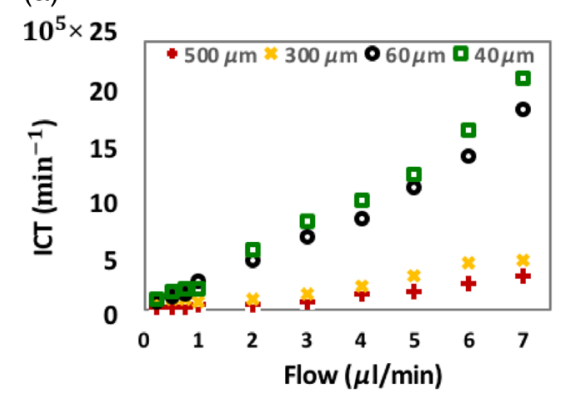

(b)

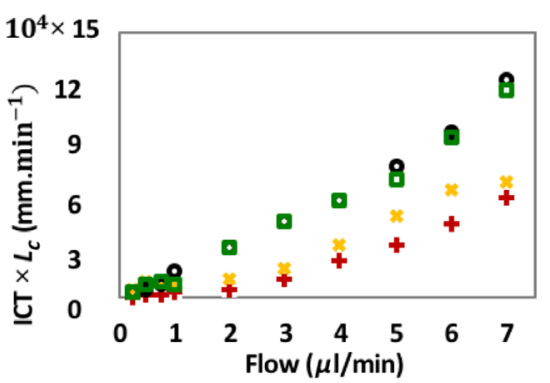

Fig. 4 Normalization by the characteristic dimension of the channels fails for large diameters. (a) Raw ICT and (b) normalized ICT. Data have been obtained by flowing $2 \%$ Intralipid solution into the channels of chip\#1. 
(a)

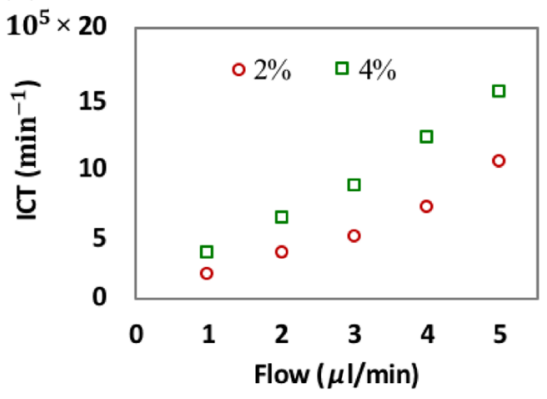

(b)

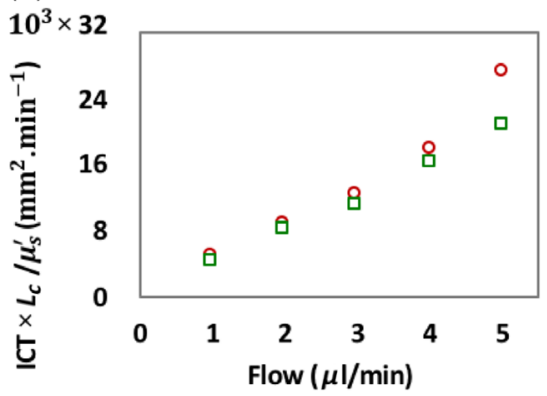

Fig. 5 Impact of the scattering properties of the flowing fluid. (a) Raw ICT and (b) normalized ICT. Data have been obtained by flowing $2 \%$ and $4 \%$ Intralipid solution into the $60-\mu \mathrm{m}$ channel of chip\#1.

(a) $10^{5} x$

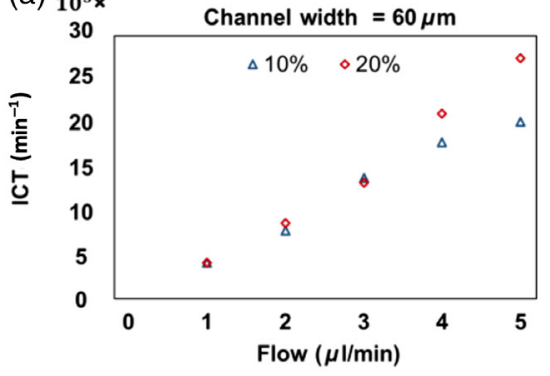

(b)

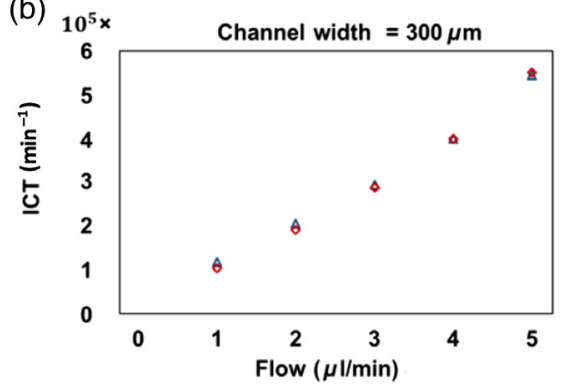

Fig. $610 \%$ and 20\% Intralipid solutions lead to ICT saturation before normalization in both 60- and $300-\mu$ m channels. (a) Raw ICT for $10 \%$ and $20 \%$ Intralipid solutions in a $60-\mu$ m channel and (b) raw ICT for $10 \%$ and $20 \%$ Intralipid solutions in a $300-\mu \mathrm{m}$ channel.

before they are backscattered is not modified when increasing the Intralipid relative concentration from $10 \%$ to $20 \%$. Such a high concentration of scatterers is not representative of blood normal values, so this does not preclude the use of speckle in blood flow imaging. However, it implies that calibration experiments of speckle imaging instruments should be performed with fluids that closely mimic the optical properties of physiological blood. Normalization of in vivo data on the basis of hematocrit values is not straightforward since the hematocrit varies within vasculature so a single value obtained by a blood sample can only be used as a general indicator. The mapping in real time of the in vivo local optical properties with a spatial resolution similar to speckle contrast could allow an accurate normalization, considering physiological hematocrit changes. Methods based on spatially modulated imaging could provide in vivo maps of the optical coefficients. ${ }^{39,40}$ The normalization to the optical scattering properties may be particularly relevant for the comparison of flow between healthy and pathological states in small animals where the hematocrit is subjected to abnormal changes. For all vessels considered, the normalization is effective for flows below $3 \mu \mathrm{l} / \mathrm{min}$ but fails with higher flows. Again this does not prevent accurate speckle flow index measurement in small animals but should be considered if speckle imaging is considered for clinical applications in humans.

\subsection{Choice of the Processing Algorithm for the Calculation of the Speckle Contrast}

In order to evaluate the influence of the processing algorithm of the raw data on the speckle contrast evaluation and the ability of the MESI approach to account for the contribution of static scatterers, we have carried out measurements on chip \#2. First, we have tested four approaches proposed previously to calculate the speckle contrast: spatial contrast $K_{\mathrm{s}}$, temporal contrast $K_{\mathrm{t}}$, spatio-temporal $K_{\mathrm{st}}$, and the temporal-spatial $K_{\mathrm{ts}}$. Experiments were carried out in chip \#2 with a transparent PDMS cover. Figure 7 shows the results of the four processing approaches of dynamic speckle contrast derivation. The temporal analysis $\left(K_{\mathrm{t}}\right)$ approach leads to high noise in all the image regions (inside and outside of the channels) due to the absence of spatial averaging. This approach fails to extract accurately the smaller channels. Using the classical spatial analysis $\left(K_{\mathrm{s}}\right)$, the contrast maps are less noisy than for $K_{\mathrm{t}}$ at the expense a coarser temporal resolution. The profile across the channels shows edges' effects around the walls of the channels due to spatial averaging. Some variabilities are observed in the contrast values inside the channels, especially for the 50- $\mu \mathrm{m}$-diameter channel. The spatiotemporal and temporo-spatial approaches $\left(K_{\mathrm{st}}\right.$ and $\left.K_{\mathrm{ts}}\right)$ provide less noisy contrast maps and demonstrate good visibility for imaging microvessels. Noticeably, both approaches result in the same contrast values at the center of all the channels [Fig. 7(b)]. The ratio of the speckle contrast inside and outside the channel is higher for the $K_{\mathrm{ts}}$ approach, especially in the larger vessel. For the $K_{\text {st }}$ analysis, spatial averaging produces strong edge effects on the channel walls, similar to the $K_{\mathrm{s}}$ approach. This does not prevent accurate speckle flow measurement since a centerline along the channel direction is used as a region of interest for flow measurements. Even the edge effects enhance the visibility and the practical location of the smaller vessels on the contrast images. Since the raw images are natively a collection of spatial frames, it is practically more efficient to perform the $K_{\text {st }}$ calculation rather than the $K_{\mathrm{ts}}$ analysis. Consequently, the $K_{\mathrm{st}}$ approach was chosen in the subsequent experiments. 
(a)

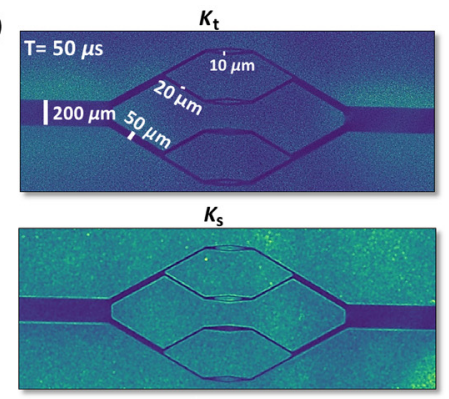

$\kappa_{\mathrm{ts}}$
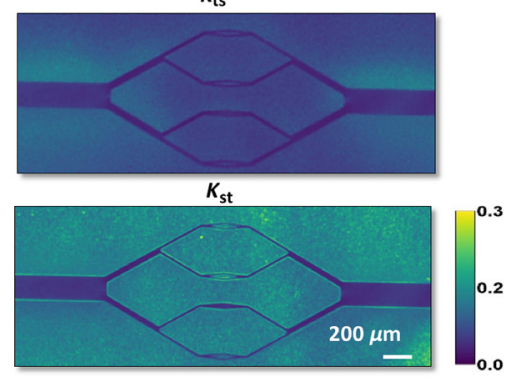

(b)

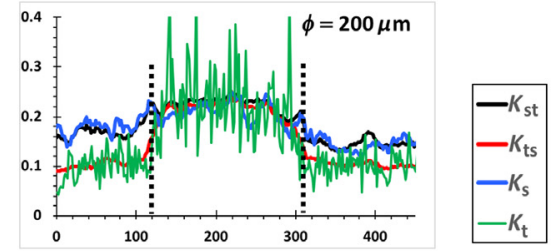

$\boldsymbol{K}$

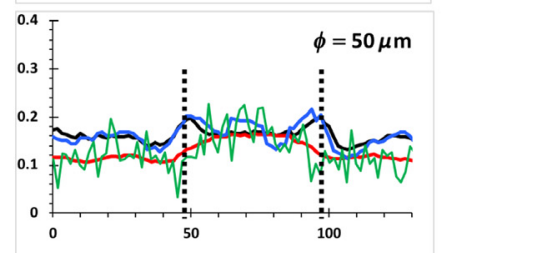

K

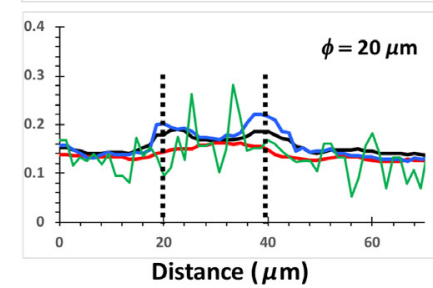

Fig. 7 Impact of the contrast processing algorithm: (a) evaluation of speckle contrast for laser speckle contrast images of microfluidic phantom chip\#2, calculated as $K_{\mathrm{t}}, K_{\mathrm{s}}, K_{\mathrm{ts}}$, and $K_{\mathrm{st}}$. (b) Plot profile corresponding to the white arrows in (a) for channels widths of 20,50 , and $200 \mu \mathrm{m}$. Dotted lines correspond to the channel walls.

\subsection{Implementation of the Data Analysis Workflow}

Three programming languages, i.e., MATLAB, Python, and $\mathrm{C}++$, were evaluated to calculate the ICT maps using the $K_{\mathrm{st}}$ contrasts stacks. Table 2 shows the comparison in terms of execution time between the three codes following the procedure described in Fig. 2 for 6 and 16 exposure times per stack $S$ and three different image sizes $(100 \times 100,300 \times 300$, and $500 \times$ 500). For in vivo experiments, numerous stacks are recorded to follow the variation of ICT values during a stimulation trial. Consequently, we also compare the processing time for a single stack (ME-1) and for 40 stacks acquisition (ME-40) corresponding to a complete single-trial acquisition. The obtained computing times indicate that $\mathrm{C}++$ analysis program is about 2 to 3 times faster than MATLAB or Python programs. Moreover, thanks to multi-CPU parallelizing, no additional time is necessary to process the 40 multiexposure stacks for the $\mathrm{C}++$ approach. Finally, this optimal procedure is able to reduce the analysis time of a full acquisition to a few minutes, compared to several hours for MATLAB or Python approaches.

\subsection{Optimization of MESI Methods for In Vivo Imaging of Dynamic Cerebral Blood Flow Changes}

The MESI model provides accurate estimation of the correlation times $\tau_{c}$ in the presence of mobile and static scatterers, as is the case for in vivo imaging. This accuracy compared to the singleexposure approach is obtained at the expense of a more complex instrumentation and data analysis. In this context, we have worked toward two goals: first, the reduction of the number of exposure times required to estimate $\tau_{c}$ and second, the optimization of the data analysis workflow. In order to estimate the correct number and values of exposure times, we have followed an empirical approach based on MESI data acquired in vivo at 16 different exposure times from $50 \mu \mathrm{s}$ up to $60 \mathrm{~ms}$. For the unbiased acquisition of MESI data, the same level of light intensity on the camera sensor should be maintained throughout the whole exposure ranges. This is possible thanks to the use of a high-power laser diode $(200 \mathrm{~mW})$ associated with an AOM that modulates simultaneously the duration and the intensity of the light pulses, whereas the sCMOS camera takes images at a fixed exposure and frequency. Figure 8(a) shows representative speckle contrast images of cortical tissues obtained in anesthetized mice for different exposure times. We have processed 32 ROIs obtained on 4 different mice with different combinations of exposures times. We found that the combination of contrast images acquired with the 6 following exposure time at $50 \mu \mathrm{s}, 200 \mu \mathrm{s}, 800 \mu \mathrm{s}, 5 \mathrm{~ms}, 20 \mathrm{~ms}$, and $60 \mathrm{~ms}$ gives the best estimates of $\tau_{c}$ in comparison to the estimate obtained using the 16 exposure times considered as a reference [Fig. 8(b)]. These 6 values are not identical but very close to the set of values $(50 \mu \mathrm{s}, 250 \mu \mathrm{s}, 750 \mu \mathrm{s}, 5 \mathrm{~ms}, 25 \mathrm{~ms}$, and $80 \mathrm{~ms})$ determined previously in a systematic approach. ${ }^{41}$ The values span the range of exposure from very short exposure up to long-exposure times, which supports the speckle model of short-exposure time being more sensitive to fast flows in arteriole, whereas longer exposure time being able to capture lower flows in the parenchyma. The longest exposure time in our set of parameters is $60 \mathrm{~ms}$ thus the frame rate of the sCMOS camera is $11.3 \mathrm{fps}$ and the temporal resolution of the MESI imaging with 6 exposure times is $528 \mathrm{~ms}$. This temporal resolution is adequate for the study of hemodynamic changes, which occur in the brain following functional activation.

The MESI model allows accounting for the contribution of static scatterers to the speckle patterns. This is of particular importance for in vivo imaging as it could allow longitudinal minimally invasive imaging. We have first carried out an analysis of speckle patterns for flows recorded in vitro on the chips \#2a,b,c sealed with 300- $\mu$ m thick PDMS covers with increasing reduced scattering coefficients. The impact of the optical scattering of the PDMS cover material on the profile of the contrast computed at the centerline section of the $20-, 50-$, and $200-\mu \mathrm{m}$ 
(a)

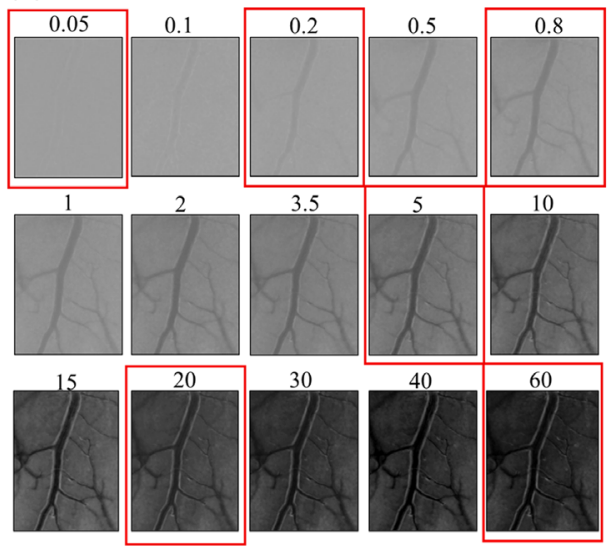

(b)

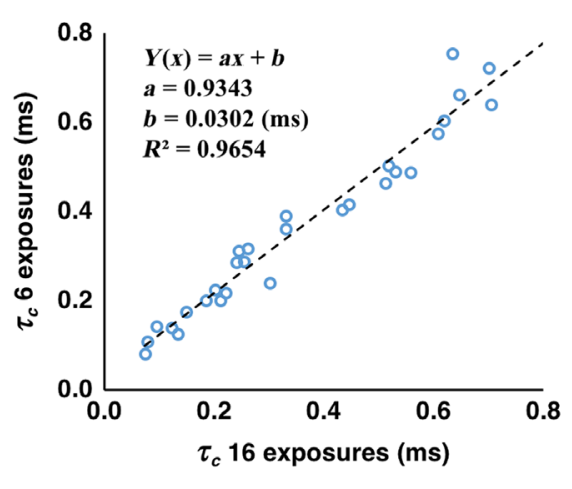

Fig. 8 Optimization of exposure times for MESI acquisition in vivo in the mouse barrel cortex. (a) Representative speckle contrast images obtained for increasing exposure times. (b) Correlation times derived using the MESI model with a set of 6 exposures times [corresponding to maps framed in red in Fig. 8(a)] as a function of correction times derived using 16 exposure times.

Table 2 Comparison of the consumed time by processing algorithms (MATLAB versus Python and $\mathrm{C}++$ ). $S$ is the number of multiexposures stacks $\left(N_{f}=S \times t\right)$.

\begin{tabular}{|c|c|c|c|c|c|}
\hline \multicolumn{3}{|c|}{ Processing time are expressed in seconds } & \multicolumn{3}{|c|}{ Image size (pixels) } \\
\hline Programs & \# of stacks & \# of exposures & $100 \times 100$ & $300 \times 300$ & $500 \times 500$ \\
\hline \multirow[t]{4}{*}{ MATLAB } & ME-1 & 6 & 26.2 & 207.9 & 550.9 \\
\hline & & 16 & 27.5 & 220.2 & 592.1 \\
\hline & ME-S $(S=40)$ & 6 & 1048.5 & 8315.7 & 22036.1 \\
\hline & & 16 & 1099.2 & 8808.4 & 23684.4 \\
\hline \multirow[t]{4}{*}{ Python } & ME-1 & 6 & 30.2 & 277.1 & 802.8 \\
\hline & & 16 & 35.7 & 300.3 & 812.7 \\
\hline & ME-S $(S=40)$ & 6 & 1208.2 & 11083.4 & 32112.0 \\
\hline & & 16 & 1427.9 & 12010.4 & 32508.1 \\
\hline \multirow[t]{4}{*}{$\mathrm{C}++$} & ME-1 & 6 & 10.8 & 97.3 & 262.5 \\
\hline & & 16 & 11.2 & 103.0 & 265.6 \\
\hline & ME- $S(S=40)$ & 6 & 10.8 & 97.3 & 262.5 \\
\hline & & 16 & 11.2 & 103.0 & 265.6 \\
\hline
\end{tabular}

diameters channels is shown in Fig. 9. The increase of the concentration of static scatterers in the PDMS cover leads to an increase of the speckle contrast values outside and inside the channels. These results arise from the sum of the contribution of the static and moving scatterers to the speckle contrast. Table 3 shows the results of the MESI analysis for three channels in the chips\#2 a,b,c. The decrease of $\rho$ values with the increase of the static scatterers concentration reflects the lower fraction of the collected light that is scattered by the moving scatterers. The correlation times and subsequent ICT values derived from the MESI model are only slightly modified by the presence static scatterers showing the robustness of the technique to account for the static scatterers contribution to the speckle contrast. The MESI model provides accurate correlation times and corresponding ICT estimations even in the presence of thin highly scattering layers like skull. This supports the use of the MESI technique for in vivo imaging in intact or thinned skull preparations.

In order to validate these findings for biospeckles recordings, we have tested the MESI technique in vivo with the intact skull and with an open craniotomy preparation in the same anesthetized mice. Figure 10(a) shows representative raw and speckle contrast images for both configurations in the same animal. The corresponding speckle contrast values as a function of exposure times are presented in Fig. 10(b). The profile of the speckle contrast varies significantly across the selected ROIs. 


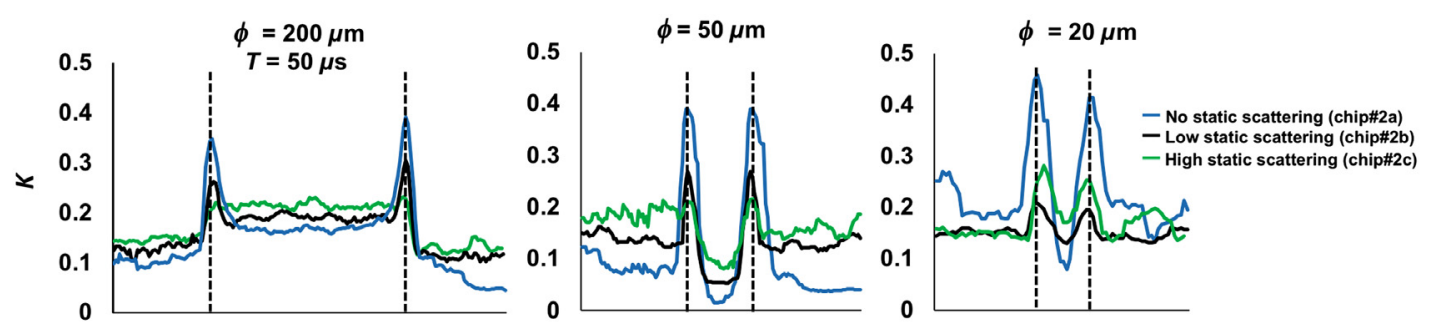

Fig. 9 Effect of static scatterers on speckle contrast values in centerline section of channels. Profile of the speckle contrast across the 20-, 50-, and 200- $\mu \mathrm{m}$ channels. Blue lines correspond to chip\#2a without static scattering. Green lines correspond to chip\#2b with low-static scattering. Black lines correspond to chip\#2c with high-static scattering. Dotted lines indicate the walls of the channels.

Table 3 Effect of static scatterers on speckle flow measurements in different channels. $\tau_{c}$ and raw ICT (not normalized) obtained on the channels of chip \#2a,b,c while flowing Intralipid-2\%. $\tau_{c}$ and ICT are not normalized for the channel diameters.

\begin{tabular}{llccc}
$Q=1 \mathrm{ml} / \mathrm{min}$ & & Chip \#2a & Chip \#2b & Chip \#2c \\
\hline$D=200 \mu \mathrm{m}$ & $\tau_{c}(\mathrm{~ms})$ & 0.0142 & 0.0128 & 0.0136 \\
& ICT & 70.53 & 78.25 & 73.74 \\
& $\rho$ & 0.8 & 0.76 & 0.42 \\
$D=50 \mu \mathrm{m}$ & $\tau_{C}(\mathrm{~ms})$ & 0.0105 & 0.0103 & 0.0108 \\
& ICT & 95.3 & 97 & 92.3 \\
& $\rho$ & 0.78 & 0.57 & 0.4 \\
$D=20 \mu \mathrm{m}$ & $\tau_{C}(\mathrm{~ms})$ & 0.0053 & 0.0058 & 0.0061 \\
& ICT & 187 & 172 & 165 \\
& $\rho$ & 0.36 & 0.33 & 0.3 \\
\hline
\end{tabular}

As expected for all ROIs, the contrast decreases with increasing exposure times. The derivation of $\tau_{c}$ and $\rho$ from the MESI model shows that the lowest $\tau_{c}$ are found in large vessels (ROI\#1) corresponding to higher flow compared to parenchyma regions with highest $\tau_{c}$ and lower flow (ROI\#3). For region \#1 and $\# 2$, the parameter $\rho$ retrieved from the MESI model in the open craniotomy is much higher compared to the same parameter derived from images obtained through the skull. This supports that $\rho$ is strongly related to the contribution of mobile scatterers. For region \#3, which we identified as parenchyma, there is no significant difference in $\rho$ before and after the craniotomy. This surprising result may arise from the fact that the proportion of moving scatterers is fairly low and that contributing static scatterers in this region are not mainly scatterers from the skull but rather from the tissue itself. In addition, the speckle patterns from this ROI originate from several capillaries with multidirectional flows, thus making the MESI data difficult to interpret. Finally, in order to estimate the accuracy of speckle imaging through skull with the MESI technique, we have considered 47 ROIs in 5 mice and compared the correlation times derived before and after the craniotomy. As shown in Fig. 10(c), there is a strong linear correlation between $\tau_{c}$ derived for both configurations. These results support previous findings carried out in a partial-craniotomy configuration. ${ }^{42}$ MESI imaging can monitor $\mathrm{CBF}$ in mice through the skull. However, these data are obtained in young mice (aged 4 to 8 weeks), and we failed to obtained consistent ICT maps for mice older than 11 weeks. In our hands, this is a practical limitation of through-skull imaging that prevent from longitudinal imaging. Long-term imaging can still be carried out using a chronic cranial window, which is feasible but difficult to practically implement over months. ${ }^{11,42}$

\subsection{MESI versus Single-Exposure Speckle Imaging of Functional Activation}

Studying local regulation of $\mathrm{CBF}$ is essential for further understanding of basic brain function as well as pathologies such as stroke. Here, to assess the ability of the MESI technique to study the relative changes in $\mathrm{CBF}$ during functional activation, we have acquired in vivo data in the $\mathrm{OB}$ and $\mathrm{BC}$ in anesthetized mice before and after sensory stimulation. Previous speckle data have been obtained during functional activation in mice brain mostly during electrical stimulation of the somatosensory cortex. ${ }^{43-47}$ They have been recorded using the single-exposure approach, which shows a limited accuracy since (i) it does not account for static scatterers and (ii) the ICT relative changes depend strongly on the single-exposure time chosen. Here we provide MESI images of $\mathrm{BC}$ and $\mathrm{OB}$ at rest and following sensory activation. To our knowledge, only a few studies have implemented single-exposure laser speckle imaging in these brain structures during physiologically relevant sensory activation. ${ }^{45,48}$ Figure 11 (a) compares the raw ICT maps derived from the same experiment using single-exposure speckle analysis at 0.8 and $5 \mathrm{~ms}$ and the ICT maps derived from the MESI analysis (using six exposures). MESI-derived ICT map shows higher contrast although surface blood vessels can already be clearly seen using the single-exposure approach. The main difference between single-exposure speckle and MESI here is that MESI provides bias free estimation by considering noises and the contribution of static scatterers. In the single-exposure approach, the ICT relative changes following activation are strongly dependent on the exposure time. The bias free approach of MESI leads to significantly higher estimation of ICT changes between rest and activation in large and small vessels [Figs. 11(c) and 11(d)].

\subsection{MESI Imaging of Functional Activation}

We have recorded MESI data during the functional activation of the $\mathrm{OB}$ and of the BC. Both models use relevant physiological activation, respectively, using puffs of odorant with controlled pure odorant molecule concentration and mechanically sustained stimulation of the whiskers. Both structures present a highly organized architecture composed of functional structures 
(a)

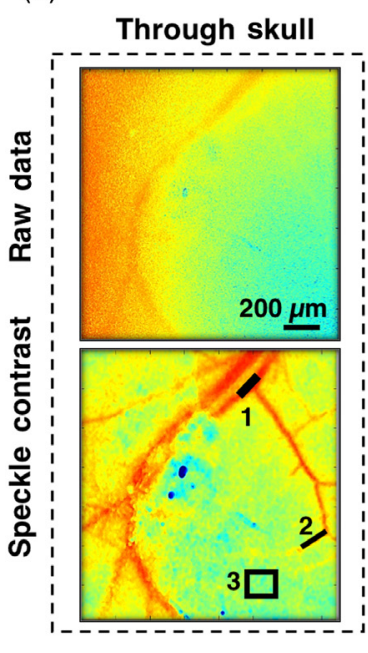

After craniotomy

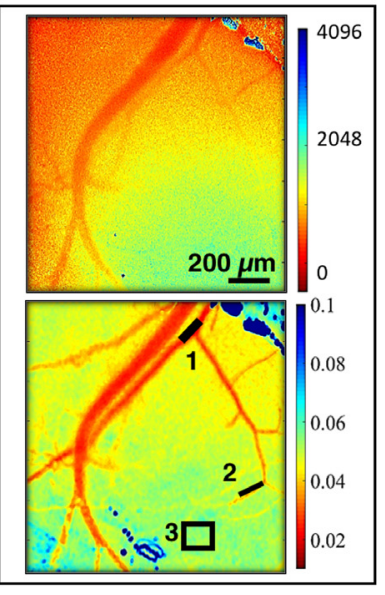

(b)

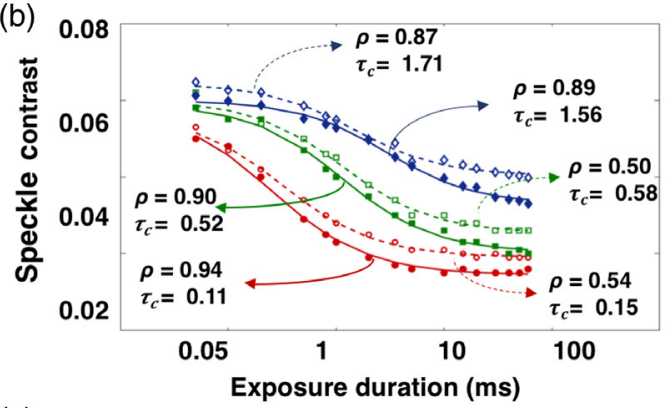

(c)

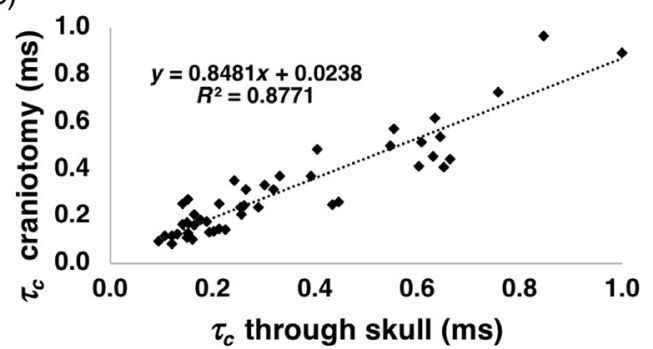

Fig. 10 Comparison of MESI imaging of the cortical tissues through skull, versus in the open craniotomy configuration in vivo in the mouse barrel cortex. (a) Comparison of raw data (pixel intensity in gray levels) and speckle contrast $K_{\text {st }}$. Numbers and bars on the contrast images correspond to ROI used to derive the $\rho$ and $\tau_{c}$ parameters in Fig. 10(b). (b) Speckle contrast as a function of the exposure time and fit to the MESI model. Open markers and dotted lines correspond to through-skull imaging. Filled markers and plain lines correspond to imaging after craniotomy. Red circles and lines = ROI\#1; green squares and lines = ROI\#2; blue diamonds and lines = ROI\#3. (c) Correlation between $\tau_{c}$ obtained in the through-skull and open craniotomy configurations in $47 \mathrm{ROIs}$ in 5 mice aged between 4 and 8 weeks.

(a)
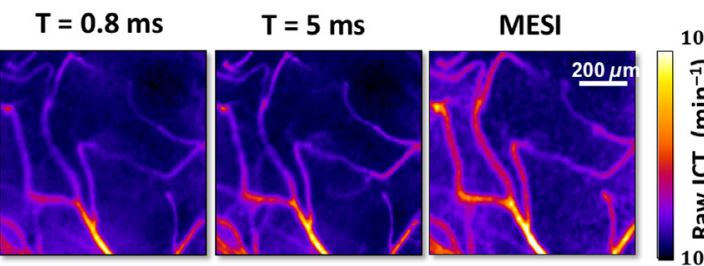

(c)

\begin{tabular}{|c|c|c|}
\cline { 2 - 3 } \multicolumn{1}{c|}{} & \multicolumn{2}{|c|}{$\%$ of raw ICT change (SD) } \\
\cline { 2 - 3 } & $\begin{array}{c}\text { Large vessels } \\
\Phi>30 \mu \mathrm{m}\end{array}$ & $\begin{array}{c}\text { Small vessels } \\
\phi<30 \mu \mathrm{m}\end{array}$ \\
\hline $\begin{array}{c}\text { MESI } \\
\text { (6 exposure times) }\end{array}$ & $25.0(3.4)$ & $16.2(2.1)$ \\
\hline $\mathrm{T}=5 \mathrm{~ms}$ & $14.5(3.8)$ & $8.0(2.2)$ \\
\hline $\mathrm{T}=0.8 \mathrm{~ms}$ & $7.3(2.9)$ & $3.4(0.6)$ \\
\hline
\end{tabular}

(b)

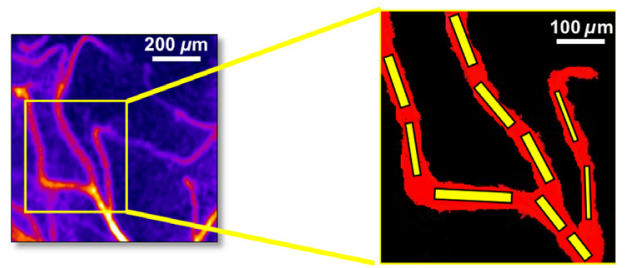

(d)

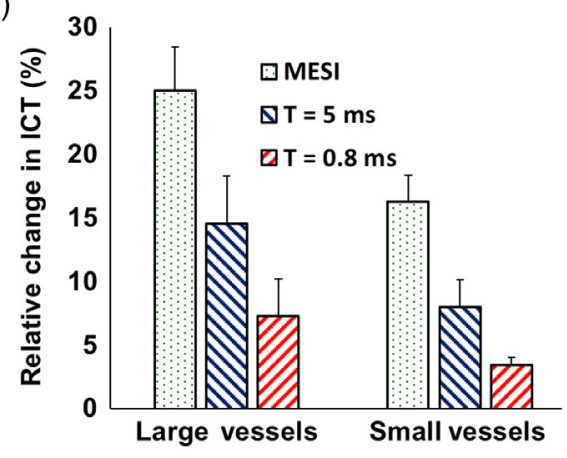

Fig. $11 \mathrm{MESI}$ versus single-exposure imaging of functional activation. (a) Representative ICT maps of olfactory bulb activation (single trial) obtained using single-exposure analysis at $T=0.8$ and $5 \mathrm{~ms}$ and MESI analysis of the same data. (b) Representative ROls used to study the ICT changes following functional activation. Blood vessels are segmented using an intensity threshold and ROIs are drawn manually at the center of blood vessels, excluding vessels walls. (c) Percentage of changes of ICT following functional activation using single-exposure analysis at 0.8 and $5 \mathrm{~ms}$ and MESI analysis. (d) Mean \% changes of ICT in large and small vessels using single exposure and MESI analysis. 
(a)

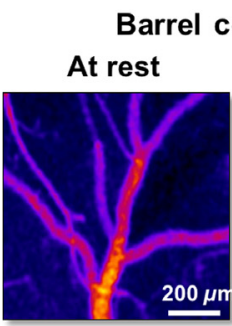

(c)

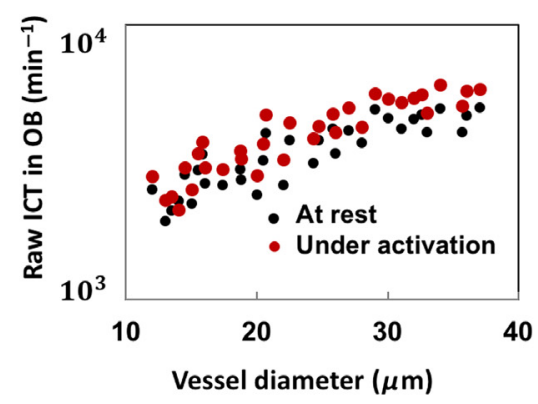

(b)

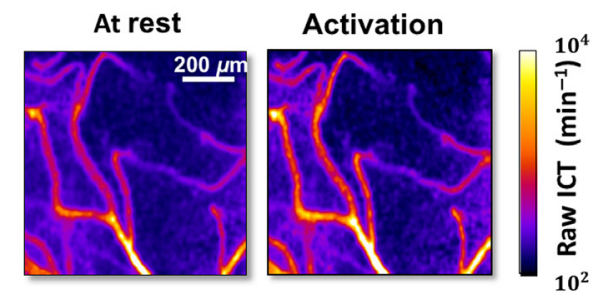

(d)

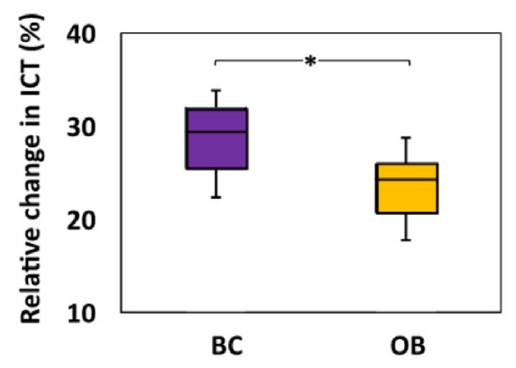

Fig. 12 In vivo MESI data obtained at rest and under functional activation in the $\mathrm{BC}$ and $\mathrm{OB}$ of anesthetized mice. (a) Representative MESI derived raw ICT maps for BC at rest and following sustained activation. (b) Representative MESI derived ICT maps for OB at rest and under activation. (c) MESI derived ICT as a function of vessel diameter for $\mathrm{OB}$ at rest (black dots) and under activation (red dots) in the same ROls. (d) Relative changes between rest and activation for ICT in BC and OB. Data in OB were obtained in 6 mice (33 ROls). Activation was a puff of air circulated on $5 \%$ diluted hexanal for $12 \mathrm{~s}$, followed by clean air with no odorant. Data in BC were obtained in 2 mice (17 ROls). Activation was studied during mechanical stimulation of whiskers at $3 \mathrm{to} 5 \mathrm{~Hz}$ for $1 \mathrm{~min}$ ). Statistical tests are unpaired student tests, $p<0.05$.

that have been shown to map sensory activation. ${ }^{49-51}$ The glomerulus is the basic functional unit in the odor map of the OB. It is a round structure of about $50-\mu \mathrm{m}$ diameter located about $100 \mu \mathrm{m}$ below the brain surface, densely packed with synapses and capillaries. In the somatosensory cortex, an individual whisker is represented in an anatomo-functional unit, the "barrel." Each module is a large ( $\sim 1$-mm in diameter $)$ six layeredneocortical structure, extending 2-mm deep (for layer 6) below the brain surface. Figures 12(a) and 12(b) show representative ICT maps for the $\mathrm{OB}$ and the $\mathrm{BC}$ at rest and following sensory stimulation. These maps show a slight but significant increase of the ICT of most of the surface vessels. Using our set up, the MESI techniques give access to vessels with diameters down to $\sim 15$ to $20 \mu \mathrm{m}$. Small vessels could be observed in the OB with a mean diameter of $25 \pm 8 \mu \mathrm{m}$, whereas larger vessels (mean diameter of $50 \pm 23 \mu \mathrm{m}$ ) were observed in the BC. Interestingly, the MESI analysis leads to similar ICT in vessels with similar diameters in both structures (data not shown). Under baseline (rest) conditions, the ratio of the average correlation times for $\mathrm{BC} / \mathrm{OB}$ in vessels with diameter from 15 to $30 \mu \mathrm{m}$ was found to be 0.894 . This shows that correlation times in vessels of the same diameter are similar in both structures, suggesting a ubiquitous relationship between diameter and blood flow index, presumably due to physical hydrodynamics rules.

We measured hemodynamic response induced by sensory stimulations in both structures over the same ROIs defined as shown in Fig. 11(b). Figure 12(c) shows the ICT values at rest and following sensory activation for single ROIs defined over the OB (6 animals, 33 ROIs). As can be seen in the bar graph of Fig. 12(d), the increase averaged on all ROIs following activation was found to be of $28 \% \pm 4 \%$ the $\mathrm{BC}$ and $23 \% \pm 3 \%$ in the OB. The distance to the upper and lower quartiles indicates that the values concentrate at the upper end of the scale for both structures. The difference of increase between BC and OB was found to be slightly significant $(p<0.05)$ but can be attributed to difference in stimulation intensities or the local morphology of the vascular network, which makes the interpretation of this difference difficult as it has many potential causes. First, the different nature and intensity of the stimulations (volatile odors versus mechanical stimulation of the whiskers) to activate these structures are very difficult to compare, but according to the literature, ${ }^{49,52}$ the sensory stimuli that we applied are moderate to strong. Second, as the blood vessels accessible in the $\mathrm{OB}$ are small arterioles and capillaries, the increase of CBF following olfactory activation is expected to be smaller than in large arteries observed in the BC. Third, neurovascular coupling mechanisms are not strictly the same in different brain regions. ${ }^{53}$ Overall, our data show that MESI is well-adapted to the study of small to moderate blood flow changes during physiologically relevant sensory stimulations. In addition, the difference in intensity of the changes between two brain sensory structures underlines the fact that the generalization of CBF changes interpretation from functional imaging should be performed cautiously according to the intensity of the stimuli and the anatomical organization of the targeted structure.

\section{Conclusion}

We have investigated the MESI technique to study functional activation in anesthetized mice. The strength of this model lies in its ability to estimate accurate correlation time independently of the static scatterers contribution. However, one of the 
assumptions of this model, i.e., single-dynamic scattering, is rarely met for in vivo imaging of rodent CBF. Our microfluidic study shows that for vessels with large diameters, normalization by the vessel diameter allows us to retrieve accurate ICT. We have also evaluated the influence of hematocrit changes. For physiologically relevant hematocrits, the data can be normalized considering the local reduced scattering coefficient, although it is difficult to achieve this for in vivo data. We have carried out MESI imaging of the $\mathrm{OB}$ and $\mathrm{BC}$ at rest and following sensory activation. The comparison of $\mathrm{BC}$ and $\mathrm{OB}$ data obtained after craniotomy showed significant differences in ICT values at rest or in an activated state indicating that the interpretation of functional imaging should carefully consider local morphological and functional specificity.

\section{Disclosures}

No conflicts of interest, financial or otherwise, are declared by the authors.

\section{Acknowledgments}

We thank A. Liège from IMNC Laboratory for valuable help on the configuration and set up of the software for the remote data analysis. This project was supported by the French Ministry of Education and Research (MESR PhD grant of H. Soleimanzad). Animal studies: All experiments were performed in accordance with the European Directive 86/609/EEC regarding the care and use of laboratory animals.

\section{References}

1. D. Briers et al., "Laser speckle contrast imaging: theoretical and practical limitations," J. Biomed. Opt. 18(6), 066018 (2013).

2. S. M. S. Kazmi et al., "Expanding applications, accuracy, and interpretation of laser speckle contrast imaging of cerebral blood flow," J. Cereb. Blood Flow Metab. 35(7), 1076-1084 (2015).

3. P. G. Vaz et al., "Laser speckle imaging to monitor microvascular blood flow: a review," IEEE Rev. Biomed. Eng. 9, 106-120 (2016).

4. J. D. Briers and A. F. Fercher, "Retinal blood-flow visualization by means of laser speckle photography," Invest. Ophthalmol. Visual Sci. 22(2), 255-259 (1982).

5. H. Cheng, Y. Yan, and T. Q. Duong, "Temporal statistical analysis of laser speckle images and its application to retinal blood-flow imaging," Opt. Express 16, 10214-10219 (2008).

6. A. Ponticorvo et al., "Laser speckle contrast imaging of blood flow in rat retinas using an endoscope," J. Biomed. Opt. 18(9), 090501 (2013).

7. A. K. Dunn et al., "Dynamic imaging of cerebral blood flow using laser speckle," J. Cereb. Blood Flow Metab. 21(3), 195-201 (2001).

8. N. Li et al., "High spatiotemporal resolution imaging of the neurovascular response to electrical stimulation of rat peripheral trigeminal nerve as revealed by in vivo temporal laser speckle contrast," J. Neurosci. Methods 176(2), 230-236 (2009).

9. P. B. Jones et al., "Simultaneous multispectral reflectance imaging and laser speckle flowmetry of cerebral blood flow and oxygen metabolism in focal cerebral ischemia," J. Biomed. Opt. 13(4), 044007 (2008).

10. K Swanul et al., "Measurements and modeling of transient blood flow perturbations induced by brief somatosensory stimulation," Open Neuroimaging J. 5, 96-104 (2011).

11. P. Miao et al., "Chronic wide-field imaging of brain hemodynamics in behaving animals," Biomed. Opt. Express 8(1), 436-445 (2017).

12. Z. Qiu et al., "A new method for evaluating regional cerebral blood flow changes: laser speckle contrast imaging in a C57BL/6J mouse model of photothrombotic ischemia," J. Huazhong Univ. Sci. Technol. Med. Sci. 36(2), 174-180 (2016).

13. L. M. Richards et al., "Low-cost laser speckle contrast imaging of blood flow using a webcam," Biomed. Opt. Express 4(10), 2269-2283 (2013).
14. A. Ponticorvo and A. K. Dunn, "How to build a laser speckle contrast imaging (LSCI) system to monitor blood flow," J. Visualized Exp. 45, 2004 (2010).

15. J. D. Briers, "Laser Doppler, speckle and related techniques for blood perfusion mapping and imaging," Physiol. Meas. 22(4), R35-R66 (2001).

16. K. Murari et al., "Contrast-enhanced imaging of cerebral vasculature with laser speckle,” Appl. Opt. 46(22), 5340-5346 (2007).

17. H. Cheng et al., "Modified laser speckle imaging method with improved spatial resolution," J. Biomed. Opt. 8(3), 559-564 (2003).

18. P. Li et al., "Imaging cerebral blood flow through the intact rat skull with temporal laser speckle imaging," Opt. Lett. 31(12), 1824-1826 (2006).

19. J. Qiu et al., "Spatiotemporal laser speckle contrast analysis for blood flow imaging with maximized speckle contrast," J. Biomed. Opt. 15(1), 016003 (2010).

20. J. W. Goodman, "Statistical properties of laser speckle patterns," in Laser Speckle and Related Phenomena, Springer, Berlin, Heidelberg, pp 9-75 (1975).

21. A. B. Parthasarathy et al., "Robust flow measurement with multiexposure speckle imaging," Opt. Express 16(3), 1975-1989 (2008).

22. P. Zakharov et al., "Quantitative modeling of laser speckle imaging," Opt. Lett. 31(23), 3465-3467 (2006).

23. E. H. Ratzlaff and A. Grinvald, "A tandem-lens epifluorescence macroscope: hundred-fold brightness advantage for wide-field imaging," J. Neurosci. Methods 36(2-3), 127-137 (1991).

24. H. Soleimanzad, H. Gurden, and F. Pain, "Development of a multiexposure speckle imaging for mice brain imaging," in Neural Imaging and Sensing, Q. Luo and J. Ding (Eds)., SPIE, Bellingham, Washington, p. UNSP 1005110 (2017).

25. J. Senarathna et al., "Laser speckle contrast imaging: theory, instrumentation and applications," IEEE Rev. Biomed. Eng. 6, 99-110 (2013).

26. ITK, "Segmentation \& Registration Toolkit," https://itk.org/ITK/ project/project.html (accessed 26 July 2018).

27. ALGLIB, "C++/C\# numerical analysis library," https://www.alglib.net/ (accessed 26 July 2018).

28. F. Ayers et al., "Fabrication and characterization of silicone-based tissue phantoms with tunable optical properties in the visible and near infrared domain," Proc. SPIE 6870, 687007 (2008).

29. J. W. Pickering et al., "Double-integrating-sphere system for measuring the optical properties of tissue," Appl. Opt. 32(4), 399-410 (1993).

30. H. Soleimanzad, H. Gurden, and F. Pain, "Optical properties of mice skull bone in the 455- to 705-nm range," J. Biomed. Opt. 22(1), 049802 (2017).

31. R. Chery et al., "Imaging odor-evoked activities in the mouse olfactory bulb using optical reflectance and autofluorescence signals," J. Visualized Exp. (56), 3336 (2011).

32. P. Miao et al., "Local scattering property scales flow speed estimation in laser speckle contrast imaging," Laser Phys. Lett. 12, 075601 (2015).

33. D. A. Boas and A. G. Yodh, "Spatially varying dynamical properties of turbid media probed with diffusing temporal light correlation," J. Opt. Soc. Am. A 14(1), 192-215 (1997).

34. S. M. S. Kazmi et al., "Flux or speed? Examining speckle contrast imaging of vascular flows," Biomed. Opt. Express 6(7), 2588-2608 (2015).

35. L. M. Richards et al., "Intraoperative multi-exposure speckle imaging of cerebral blood flow," J. Cereb. Blood Flow Metab. 37(9), 3097-3109 (2017).

36. N. Bosschaart et al., "A literature review and novel theoretical approach on the optical properties of whole blood," Lasers Med. Sci. 29(2), 453-479 (2014).

37. R. Michels, F. Foschum, and A. Kienle, "Optical properties of fat emulsions," Opt. Express 16(8), 5907-5925 (2008).

38. K. Khaksari and S. J. Kirkpatrick, "Combined effects of scattering and absorption on laser speckle contrast imaging," J. Biomed. Opt. 21(7), 076002 (2016).

39. K. P. Nadeau, A. J. Durkin, and B. J. Tromberg, "Advanced demodulation technique for the extraction of tissue optical properties and structural orientation contrast in the spatial frequency domain," J. Biomed. Opt. 19(5), 056013 (2014).

40. J. P. Angelo, M. van de Giessen, and S. Gioux, "Real-time endoscopic optical properties imaging," Biomed. Opt. Express 8(11), 5113-5126 (2017). 
41. S. M. S. Kazmi, S. Balial, and A. K. Dunn, "Optimization of camera exposure durations for multi-exposure speckle imaging of the microcirculation," Biomed. Opt. Express 5(7), 2157-2171 (2014).

42. C. J. Schrandt et al., "Chronic monitoring of vascular progression after ischemic stroke using multiexposure speckle imaging and two-photon fluorescence microscopy," J. Cereb. Blood Flow Metab. 35(6), 933-942 (2015).

43. A. K. Dunn et al., "Spatial extent of oxygen metabolism and hemodynamic changes during functional activation of the rat somatosensory cortex," Neuroimage 27(2), 279-290 (2005).

44. A. Devor et al., "Stimulus-induced changes in blood flow and 2-deoxyglucose uptake dissociate in ipsilateral somatosensory cortex," J. Neurosci. 28(53), 14347-14357 (2008).

45. B. Weber et al., "Optical imaging of the spatiotemporal dynamics of cerebral blood flow and oxidative metabolism in the rat barrel cortex," Eur. J. Neurosci. 20(10), 2664-2670 (2004).

46. M. A. Davis et al., "Sensitivity of laser speckle contrast imaging to flow perturbations in the cortex," Biomed. Opt. Express 7(3), 759-775 (2016).

47. W. Lau, S. Tong, and N. V. Thakor, "Spatiotemporal characteristics of low-frequency functional activation measured by laser speckle imaging," IEEE Trans. Neural Syst. Rehabil. Eng. 13(2), 179-185 (2005).

48. S. Uchida and F. Kagitani, "Effect of basal forebrain stimulation on extracellular acetylcholine release and blood flow in the olfactory bulb," J. Physiol. Sci. 68(4), 415-423 (2018).

49. C. C. H. Petersen, "The barrel cortex-integrating molecular, cellular and systems physiology," Pflugers Arch. 447(2), 126-134 (2003).

50. N. Uchida et al., "Odor maps in the mammalian olfactory bulb: domain organization and odorant structural features," Nat. Neurosci. 3(10), 1035-1043 (2000).

51. F. Pain, B. L'heureux, and H. Gurden, "Visualizing odor representation in the brain: a review of imaging techniques for the mapping of sensory activity in the olfactory glomeruli," Cell Mol. Life Sci. 68(16), 2689-2709 (2011).

52. M. Wachowiak and L. B. Cohen, "Representation of odorants by receptor neuron input to the mouse olfactory bulb," Neuron 32(4), 723-735 (2001).

53. I. M. Devonshire et al., "Neurovascular coupling is brain regiondependent," Neuroimage 59(3), 1997-2006 (2012).

Biographies of the authors are not available. 